\title{
STRUCTURAL BATTERIES MADE FROM FIBRE REINFORCED COMPOSITES
}

\author{
S. Ekstedt ${ }^{1}$, M. Wysocki ${ }^{1}$, L. E. Asp ${ }^{1,2}$ \\ ${ }^{1}$ Swerea SICOMP AB Box 104, SE-43138 Mölndal, Sweden \\ ${ }^{2}$ Luleå University of Technology, SE-97187 Luleå, Sweden \\ sofia.ekstedt@swerea.se
}

\begin{abstract}
SUMMARY
Structural, fibre reinforced, battery prototypes with two types of electrolyte matrix material, a gel and a solid polymer, have been manufactured. This was to confirm the concept of using carbon fibres as current collector in the anode as well as providing a mechanical load-carrying functionality. As a result, functioning batteries with gel electrolyte have been produced and their properties have been characterised.
\end{abstract}

Keywords: multifunctional, electrical properties, mechanical properties, polymer electrolyte, carbon fibre

\section{INTRODUCTION}

Weight is a premium on many components, and in such applications anything, which does not contribute to the load carrying capacity is structurally parasitic. The focus of this paper is one such example; batteries for electrical energy storage. The conventional design approach attempts to maximise the efficiency of individual subcomponents, focussing on their ability to perform their individual task. A different approach is to create novel multifunctional materials, which simultaneously perform more than one function, offering significant savings in system level mass and volume, or performance benefits such as improved durability or redundancy. This design approach is relatively new, and faces significant engineering design, material and application challenges.

The versatility of polymer composite materials offers an ideal opportunity to develop novel multifunctional materials which can store electrical energy required to power systems, whilst meeting the demands of the mechanical loading. Carbon fibres are particularly attractive as they are commonly used as both electrodes and a high performance structural reinforcement, but usually the forms of carbon is different. Work in the US [1,2], UK [3] and at Swerea SICOMP [4] have already demonstrated that such multifunctional materials can be synthesised.

A battery has three major parts; one anode and one cathode, and an electrolyte. The active ion, in this case lithium, is intercalated and extracted from the electrodes during charge and discharge. The electrolyte is an ion conductive medium of polymers and salt. During discharge, ions are transported through the electrolyte, from anode to cathode. Electrons are transported from the electrodes via an outer circuit. A battery with short 
ion peculation distances is preferable from electrochemical point of view. Hence, it is common practice to manufacture thin electrodes and electrolytes. This layered structure is similar to composites and therefore it is a potential basis for production of a structural composite with ability to store electrical energy. Moreover, the open circuit potential (OCP) for lithium ion battery is high and varies between $2.5-4.2 \mathrm{~V}$ leading to high electro motive force. Finally, lithium has low molecular weight $(6.94 \mathrm{~g} / \mathrm{mol})$, which results in low weight characteristics and high specific energy.

An early attempt to develop structural batteries has been performed by Wetzel and coworkers [1]. In their work, they have developed structural batteries containing solvent free electrolytes and electrodes using carbon fibres. Materials with satisfactory mechanical properties and acceptable ion conductivity were developed. The electrolytes evaluated were produced through synthesising of vinyl ester (VE) and polyethylene glycol (PEG), combining the conductive properties of PEG with mechanical stiffness of VE. The salt used in this study was lithium triflate $\left(\mathrm{CF}_{3} \mathrm{SO}_{3} \mathrm{Li}\right)$, which is the most well understood and studied system. In another study Snyder et al. [2], investigated the relationship between mechanical strength and electrical properties in polymers used as electrolytes as well as the impact of salt content. The polymers in [2] were similar to those in [1], however, the content of ethylene groups was varied. The results showed an inverse 1:1 relationship for mechanical stiffness vs. ion conductivity. Regarding the salt content it showed to have minor importance for mechanical stiffness but influenced the conductivity.

The objective of this paper is to develop different structural material combinations for use in composite batteries and to analyse their mechanical and electrical properties. This presents challenges including manufacturing, materials selection and material characterisation. Batteries produced in this study are made from carbon fibres, aluminium mesh and glass fibre to obtain good mechanical properties together with reasonable ion conductivity. Two types of electrolytes are used; one gel and one polymer matrix together with lithium iron phosphate salt $\left(\mathrm{LiFePO}_{4}\right)$.

\section{THEORETICAL MODELS}

The load carrying batteries are evaluated both regarding their mechanical stiffness and electrical characteristics. Since the batteries are to be used as multifunctional composites the properties are not on par with single functional composites.

\section{Mechanical properties}

The mechanical stiffness is evaluated using micromechanics. Rule-of-mixture together with Halpin-Tsai and laminate theory were used to calculate the mechanical properties of the composites batteries. The Halpin-Tsai is given as

$$
E_{T}=\frac{E_{m}(1+\xi \eta f)}{(1+\eta f)},
$$

where 
$\eta=\frac{\left(\frac{E_{f}}{E_{m}}-1\right)}{\left(\frac{E_{f}}{E_{m}}+\xi\right)}$,

where $E_{T}$ is the transverse stiffness of the composite, $E_{m}$ is the stiffness of matrix, $E_{f}$ is the stiffness of fibre, $f$ is the fibre fraction and $\xi$ is an adjustable parameter set to 2 to represent circular fibres.

The mechanical stiffness of each ply was calculated and related to the mechanical behaviour of the total composite. The material consists of three different types of fibres; (i) aluminium in the cathode, (ii) glass fibre in the separator and (iii) carbon fibre in the anode. Considering the different types of fibre reinforcement together with matrix properties an overall stiffness for the laminate is calculated using laminate theory.

The batteries are made of 3 weaves stacked in a laminate. In this analysis each weave is modelled symmetric and balanced. In the analyses measured volume fractions and densities were employed, the remaining material properties used were sourced in the open literature.

\section{Electrical properties}

The theoretical specific charge capacity, $Q_{s}^{\text {th }}$, is the amount of charge received from the cell and depends on the energy charge displacement [5]

$$
Q_{s}^{t h}=\frac{I t}{m}=\frac{z F}{M}
$$

where $z$ is number of charges, $F$ is Faraday's constant and $M$ is the molar mass for all participating species. The specific power energy, $W_{s}^{\text {th }}$, is given by

$W_{s}^{t h}=E_{n o m} Q_{s}^{t h}$

where $E_{\text {nom }}$ is the nominal electrical potential.

\section{MATERIALS AND EXPERIMENTAL}

The studied batteries are based on lithium ion, $\mathrm{LiFePO}_{4}$, chemistry. The layered structure of the batteries consisted of three parts. An anode, made from carbon fibre weave with a copper collector, a separator, made from glass fibre weave, and a cathode, made of $\mathrm{LiFePO}_{4}$ coated onto aluminium fibre weave used as a collector. Both solid polymer and polymer gel electrolytes were evaluated. The solid polymer electrolyte was an unspecified solid polymer electrolyte provided from a battery manufacturer. Infrared (IR) spectroscopy, however, revealed it to consist of PVDF. The gel electrolyte was made from $30 \mathrm{wt} \%$ PMMA (Mw 996000) and $70 \mathrm{wt} \%$ ethylene carbonate: dimethyl 
carbonate, 1:1, (EC:DMC) $(1 \mathrm{M} \mathrm{LiPF} 6)$. To enhance mechanical properties of the separator layer, the electrolyte mixture was applied onto a glass fibre weave.

\section{RESULTS AND DISCUSSION}

The batteries were manufactured in argon filled glovebox with low humidity, $<15 \mathrm{ppm}$, in order to protect the chemicals from $\mathrm{H}_{2} \mathrm{O}$. Heat and pressure was applied to consolidate the composite batteries, also ensuring contact between electrodes and electrolyte. The same procedure was used for both solid and gel electrolyte. After manufacturing, the structural battery was placed in an airtight bag to protect it from atmospheric humidity, see Figure 1. Four structural batteries were produced. The main idea was to investigate the possibility of producing multifunctional composites as proof of concept.

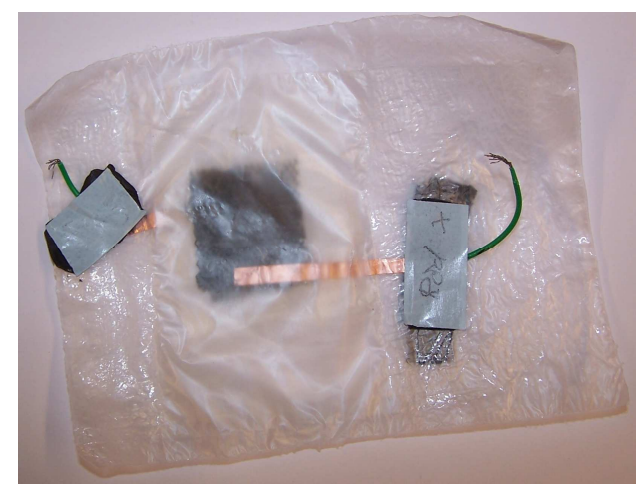

Figure 1. Manufactured battery.

\section{Mechanical properties}

Mechanical properties of the structural battery were calculated using material properties of the various laminas. Results are presented in Table 1.

Table 1. Predicted mechanical properties of the structural batteries.

\begin{tabular}{lccc}
\hline$E_{x}$ & $E_{y}$ & $v_{x y}$ & $G_{x y}$ \\
\hline $35.2 \mathrm{GPa}$ & $35.2 \mathrm{GPa}$ & 0.32 & $5.2 \mathrm{GPa}$ \\
\hline
\end{tabular}

Carbon fibres is the critical constituent with the lowest elongation to break and will therefore fail first upon loading. In this study it is assumed that this appears at a strain of 
$\varepsilon_{B}=1 \%$ giving a corresponding critical stress for the whole laminate as $\sigma_{\text {structural }}=350 \mathrm{MPa}$. The overall mechanical properties of the composites battery is compared to typical glass and carbon fibre composites in Table 2.

Table 2. Comparison between structural battery and conventional composite material stiffness[5].

\begin{tabular}{lc}
\hline Composite & $\mathrm{E}_{\mathrm{x}}(\mathrm{GPa})$ \\
\hline Structural battery & 35 \\
Glass fibre / Epoxy & 23 \\
Carbon fibre /epoxy & 75 \\
\hline
\end{tabular}

\section{Electrical properties}

Following reactions takes place in the battery:

Cathode: $\mathrm{Li}_{1-x} \mathrm{FePO}_{4}+x \mathrm{Li}^{+}+x e^{-} \rightarrow \mathrm{LiFePO}_{4}$,

Anode $: \mathrm{Li}_{x} \mathrm{C}_{6} \rightarrow \mathrm{Li}_{1-x} \mathrm{C}_{6}+x \mathrm{Li}^{+}+x e^{-}$.

The electrical properties are given by the electrochemical potentials. In particular, this battery containing $\mathrm{Li}_{\mathrm{x}} \mathrm{C}_{6}$ and $\mathrm{LiFePO}_{4}$ has an open circuit potential of

$O C P=3.3 \mathrm{~V}$.

Using densities and electrochemical properties the battery's theoretical specific charge capacity and theoretical specific power energy content are obtained. In a charge/discharge cycle experiment the nominal potential was measured to $2.3 \mathrm{~V}$. The nominal potential together with the equations (3) and (4) gives the specific charge capacity and specific power energy content as

$Q_{s}^{\text {th }}=116.6 \mathrm{Ah} / \mathrm{kg}$,

$W_{s}^{\text {th }}=268.2 \mathrm{Wh} / \mathrm{kg}$.

Compared to existing batteries the electrical properties are very good, cf. Table 2 were common batteries are compared to those predicted for the present prototypes. 
Table 3. OCP and specific energy for secondary batteries.

\begin{tabular}{lcc}
\hline Battery & OCP $(\mathrm{V})$ & $W_{s}^{\text {th }}(\mathrm{Wh} / \mathrm{kg})$ \\
\hline Lead/Acid & 2.1 & 35 \\
Lithium/Cobolt & 4.0 & 170 \\
Lithium/Manganese & 3.3 & 130 \\
Structural Battery & 3.3 & 116 \\
\hline
\end{tabular}

\section{CONCLUDING REMARKS}

In this paper structural battery prototypes are experimentally and theoretically characterised for their multifunctional abilities. Structural battery composite materials built from carbon and aluminium fibre weave electrodes, separated by a glass fibre weave, with a polymer electrolyte as matrix material have been successfully manufactured and monitored for their nominal electric potential. Detailed analyses of the structural battery mechanical and electric properties have been performed. The theoretical results from these analyses suggest that structural batteries with stiffness and in-plane strength comparable to conventional polymer composites can be achieved. Likewise, the analytical predictions of the structural battery specific charge capacity and specific power energy content indicate these materials to be very promising also from an electric point of view.

Even if a structural battery does not match the mechanical and electrical properties of the conventional polymer composite or Li-polymer battery respectively it will contribute to mass savings in an structure/energy storage system as the one employed in today's hybrid electric vehicles. The results from this study imply that significant mass savings indeed can be achieved.

\section{ACKNOWLEDGEMENTS}

Work by Tony Carlsson and Daniel Ordéus is gratefully acknowledged.

\section{References}

1. Wong EL, Baechle, DM, Xu K, Carter RH, Snyder JF, Wetzel ED, "Design and processing of structural composite batteries”, Proceedings of SAMPE 2007, Baltimore, MD, 3-7 June 2007.

2. Snyder JF, Carter RH, Wetzel ED, "Electrochemical and mechanical behaviour in mechanically robust solid polymer electrolytes for use in multifunctional structural batteries”, Chem. Mater, 2007, Maryland, April 2007.

3. Carlsson T, Ordeus D, Wysocki M, “Load carrying composites for electrical energy storage”, SICOMP TR08-002, 2008. 
4. Hamann C H, Hamnett A., Vielstich W., Electrochemistry, WILEY-VCH, $2^{\text {nd }}$ ed., Weinheim, 2007.

5. Agarwal B. D, Broutman L J, Analysis and performance of fibre composites, John Wiley \& Sons, Inc., $2^{\text {nd }}$ ed., New York, 1990. 